\title{
A FUSION METHOD OF SAR AND OPTICAL IMAGES FOR URBAN OBJECT EXTRACTION
}

\author{
Jia Yonghong ${ }^{\mathrm{a}, \mathrm{b}, \mathrm{c}}$, Rick S. Blum ${ }^{\mathrm{c}}$ \\ a School of Remote Sensing and Information Engineering, Wuhan University, Wuhan, China - yhjia2000@sina.com \\ b State Key Laboratory of Information Engineering in Surveying, Mapping and Remote Sensing, Wuhan University, \\ Wuhan, China - yhjia2000@sina.com \\ C Electrical and Computer Engineering Dept, Lehigh University, Bethlehem, PA USA - rblum@eecs.lehigh.edu
}

Commission VII, WG VII/6

KEY WORDS: Satellite remote sensing, Sharpening, Image understanding, Fusion, Land cover.

\begin{abstract}
:
A new image fusion method of SAR, Panchromatic (Pan) and multispectral (MS) data is proposed. First of all, SAR texture is extracted by ratioing the despeckled SAR image to its low pass approximation, and is used to modulate high pass details extracted from the available Pan image by means of the á trous wavelet decomposition. Then, high pass details modulated with the texture is applied to obtain the fusion product by high pass filter based on modulation (HPFM) fusion method. A set of image data including co-registered Landsat TM, ENVISAT SAR and SPOT Pan is used for the experiment. The results demonstrate accurate spectral preservation on vegetated regions, bare soil, and also on textured areas (buildings and road network) where SAR texture information enhances the fusion product, and the proposed approach is effective for image interpret and classification.
\end{abstract}

\section{INTRODUCTION}

Image fusion is capable of integrating different imagery data creating more information than that from a single sensor, and it has received tremendous attention in the remote sensing literature. Many image fusion algorithms and software tools have been developed, such as the IHS (Intensity, Hue, Saturation), PCA (Principal Components Analysis), SVR (Synthetic Variable Ratio) and wavelet based fusion[1]. However, such available algorithms are not efficient for the fusion of SAR and optical images any more. In an urban area, many land cover types/surface materials are spectrally similar. This makes it extremely difficult to analyze an urban scene using a single sensor[2][3]. Some of these features can be discriminated in a radar image based on their dielectric properties and surface roughness. The objective of our study is to present a novel image fusion method of SAR, Panchromatic (PAN) and multispectral (MS) data for urban object extraction. SAR texture is extracted by ratioing the despeckled SAR image to its low pass approximation, and is used to modulate high pass details extracted from the available Pan image by means of the á trous wavelet decomposition. High pass details modulated with the SAR texture is applied with HPFM (High Pass Filterbased Modulation) to obtain the fusion product. The following is introduction of the proposed fusion method.

\section{METHODOLOGY}

\section{1 Á trous wavelet}

Wavelet transform produces the images in different resolution. Wavelet representation refers to both spatial and frequency space. It can show a good position of an image in spatial and frequency space[4].
There are different approaches to do wavelet decomposition. One of them is Mallat algorithm which can use wavelet function such as Daubechies functions. Here we use the á trous algorithm, which uses dyadic wavelet to merge non-dyadic data in a simple and efficient procedure. In this algorithm for the discrete wavelet transform we must do the successive convolution with a filter. To convolve the image and the filter, we use convolution function directly. In each step we get a version of the image $I_{1}, I_{2}, \ldots$. The wavelet coefficient is defined as the following

$$
w c_{L}=I_{L-1}-I_{L} \quad L=1,2, \ldots, n
$$

If we decompose an image I into wavelet coefficients, then we can write 
in which $I_{r}$ is a residual image. In this approach all wavelet planes have the same number of pixels as the original image.

\subsection{SAR texture extraction}

The Lee filter for SAR speckle removal is used to reduce the effect of the multiplicative noise on homogeneous areas at first. Thus, the ratio image $\mathrm{M}$ of despeckled SAR $d s$ image to its lowpass approximation $\mathrm{SAR}_{\mathrm{L}}$, obtained by the á trous wavelet algorithm as approximation at level $L$, is computed. The ratio image $\mathrm{M}$ has unity mean value. Due to imperfect despeckling, intrinsic signal variability of rough surfaces, and instability of image ratioing, the spurious fluctuations in homogeneous areas around the mean value $m=1$ appear obviously.

By denoting with $\sigma_{t}$ the standard deviation of the distribution of the modulating SAR texture $\mathrm{M}$, a soft thresholding with threshold $\theta=k \sigma_{t}$ is applied to $M$ : Values outside the interval $\left[1-k \sigma_{t} ; 1+k \sigma_{t}\right]$ are diminished in modulus by $k \sigma_{t} t$ and retained, while values lying within this interval are set to the mean value. The resulting texture map $M_{\theta}$ contains spatial features which are easily detected by the SAR image. It can be properly modulated into wavelet planes of the panchromatic image produced by the á trous wavelet algorithm to obtain new wavelet planes.

\subsection{HPFM (high pass filter based on modulation) fusion technique}

High pass filter based on modulation fusion technique aims at preserving the spectral characteristics of the MS image by keeping the result independent of the spectral property of the higher resolution image used for modulation[5]. The HPFM processing formula for the case of the multi-spectral images sharpening by means of a co-registered high resolution Panchromatic image $\mathrm{P}$ is the following

$$
F_{k}(i, j)=M S_{k}(i, j)+K(i, j) \cdot P_{H}(i, j)
$$

where $M S_{k}$ is the resampled $k$-th band of the MS image, $P_{H}(i$, $j$ ) is high frequence details of high resolution Panchromatic image. In the proposed method, it is replaced by the wavelet planes of the panchromatic image, produced by á trous wavelet algorithm, modulated with the SAR texture. $K(i, j)$ is decided by the following forma

$$
K(i, j)=M S_{k}(i, j) / P_{L}(i, j)
$$

where $P_{L}(i, j)$ is low frequence information of high resolution Panchromatic image, and $P(i, j)=P_{L}(i, j)+P_{H}(i, j)$.

The proposed fusion scheme applied to obtain the fused image is shown in Figure. 1.

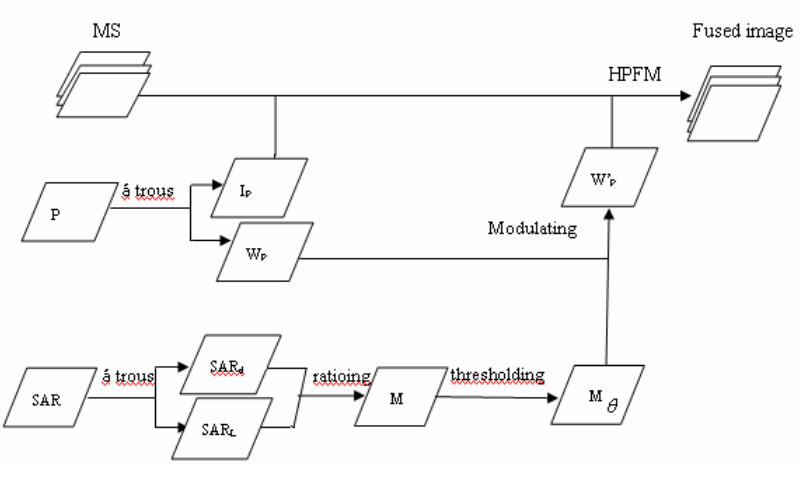

Figure. 1 The proposed fusion schedule

\section{EXPERIMENTS AND ANALYSIS}

The input dataset is composed of an ENVISAT SAR image of urban area of Wuhan, Landsat-5 TM images (band 3, 4, 5) and a SPOT Pan image. All the data have been accurately coregistered to the SPOT Pan image, as shown in Figures 2(a), 2(b) and 2(c). Texture will be blurred in the fusion image if the accuracy of co-registration among the SAR image, the TM images and the SPOT Pan image is not high enough. Figure 3 is the fused images by proposed fusion method. The fused images are evaluated in visual examination and quantitative analysis.

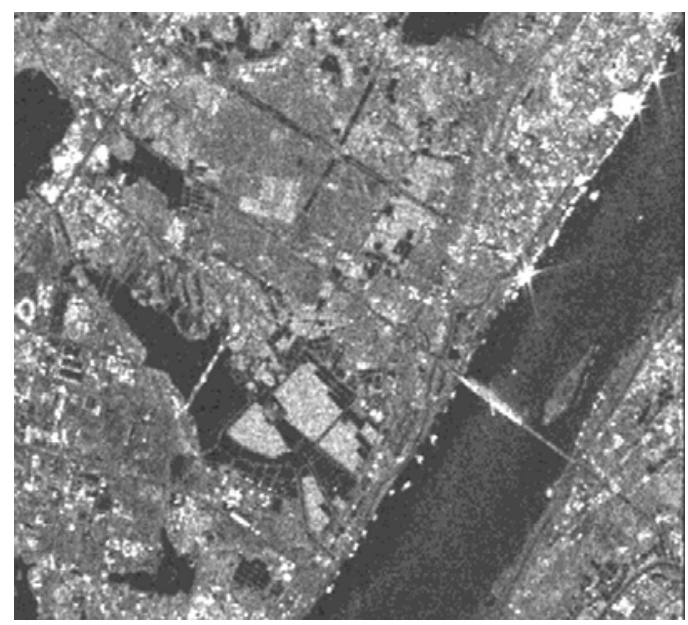

2(a) ENVISAT SAR image

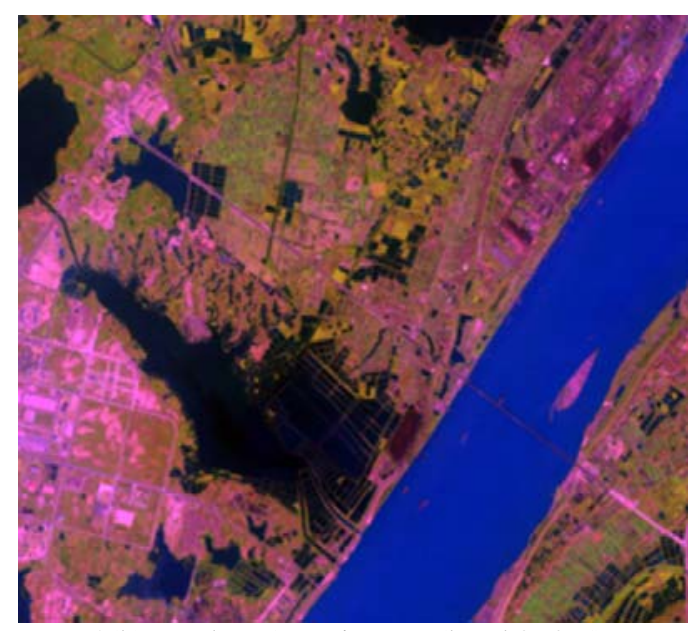

2(b) Landsat-5 TM images (band 3, 4, 5) 


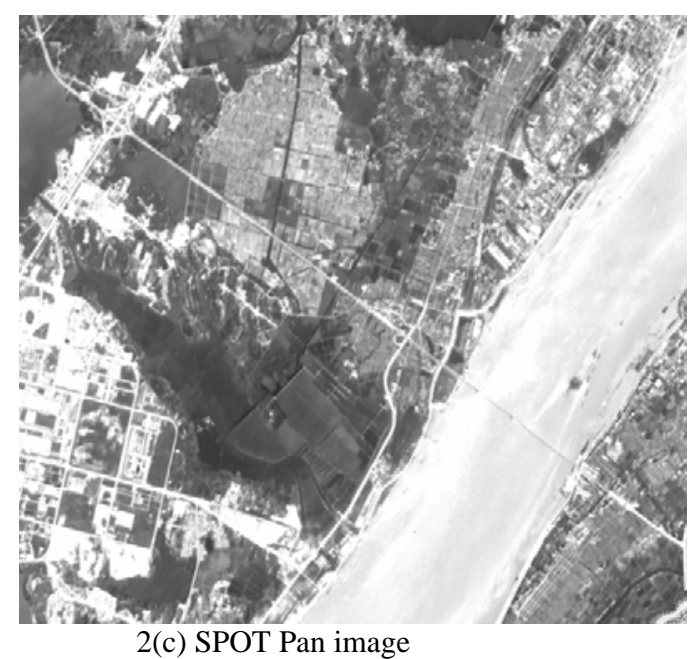

Figure 2.The co-registered images

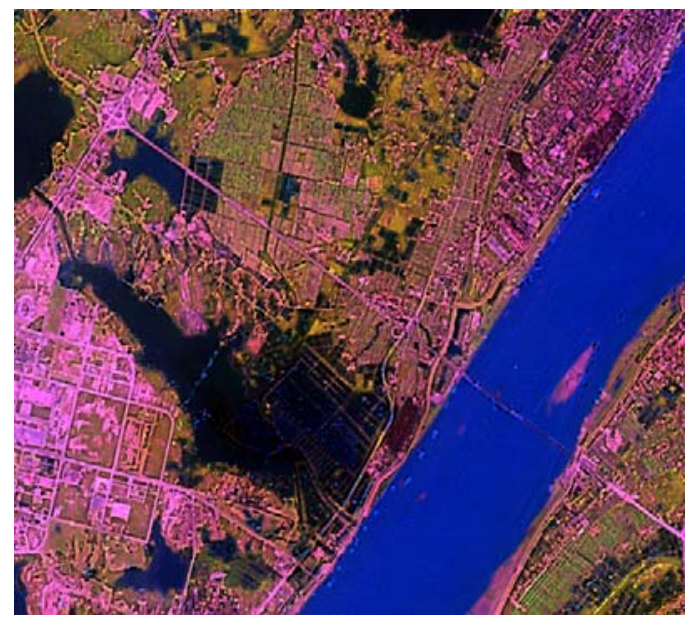

Figure 3.The fused images

\subsection{Visual examination}

Compared with from figure 2(b) and figure 3, urban and builtup areas, as well as roads are clearly enhanced and almost perfect preservation of spectral signatures is visible in the fused images. Visual examination suggests that the fused images are higher in spatial resolution than that of the Landsat TM images, and have better visual effect. Due to limitations of human vision, comparison and appreciation by visual methods does not reveal the exact potentials of the fusion methods. Hence, a comparison of image statistics is attempted to evaluate the results of fusion.

\subsection{Quantitative analysis}

In addition to visual analysis, we conduct a quantitative analysis. We base our analysis of the experimental results on entropy, average gradient of the fused images and correlation coefficient (Table 1) between the fused images and the Landsat TM images.

In Table 1, we show that the entropy and the average gradient are bigger than the corresponding values of the TM images. The correlation coefficient between the fused images and the Landsat TM images is very high. From these results, we can say that the proposed fusion method provides more detailed spatial information,simultaneously, preserves spectral content of the MS image. The proposed approach is effective for image interpret and classification. Its effectiveness for urban object extraction will be presented in the other paper.

\begin{tabular}{|l|l|l|l|}
\hline & Entropy & $\begin{array}{l}\text { Average } \\
\text { gradient }\end{array}$ & $\begin{array}{l}\text { Correlation } \\
\text { coefficient }\end{array}$ \\
\hline TM images(band & 5.40 & 9.33 & 1.0 \\
$3,4,5)$ & 5.32 & 5.90 & 1.0 \\
\hline The fused & 5.25 & 13.92 & 1.0 \\
$\begin{array}{l}\text { images } \\
\begin{array}{l}\text { By the proposed } \\
\text { method }\end{array}\end{array}$ & 5.57 & 10.02 & 0.89 \\
\hline
\end{tabular}

Table 1. Evaluation of the fused image

\section{CONCLUSION}

A new image fusion method of SAR, Panchromatic (Pan) and multispectral (MS) data is proposed. The SAR texture extraction and high pass details of Pan image modulated with the SAR texture are reasonable.The proposed method can improve spatial resolution and simultaneously preserve spectral content of the MS image ,and is effective for image interpret and classification. For future work, the authors would be looking at evolving to a more autonomous fusion system.

\section{ACKNOWLEGEMENT}

The authors would like to express their sincere gratitude to the anonymous referees for pointing out several typos and for some very helpful comments.

\section{REFERENCES}

1. L. Alparone, S. Baronti, A.Garzelli, et al,"Landsat ETM+ and SAR Image Fusion Based on Generalized Intensity Modulation, " IEEE Trans. on Geosci. Remote Sensing, vol .42, pp. 2832 - 2839, 2004.

2. B.C.Forster,“An examination of some problems and solutions in monitoring urban areas from satellite platforms , "International Journal of Remote Sensing,Vol.6, pp139-151, 1985.

3. G.F. Hepner, B. Houshmand, Kulikov,etal, "Investigation of the Integration of AVIRIS and IFSAR for urban analysis," Photogrammetric Engineering and Remote Sensing,vol.64, pp813-820,1998.

4. T. Ranchin and L.Wald,"Fusion of High Spatial and Spectral Resolution images: The ARSIS Concept and Its Implementation , "Photogrammetric Engineering and Remote Sensing,vol.66,pp.49-61,2000.

5. C. Pohl and J. L.Van Genderen,"Multisensor image fusion in remote sensing: concepts, methods and 
applications, ”International Journal of Remote Sensing, vol.19,pp.823-854, 1998. 